\title{
Limited English Proficient Patient Visits and Emergency Department Admission Rates for Ambulatory Care Sensitive Conditions in California: a Retrospective Cohort Study
}

\author{
Lucy Schulson, MD, MPH', ${ }^{1}$, Meng-Yun Lin, PhD, MPH \\ Michael K. Paasche-Orlow, MD, MA, MPH', and Amresh D. Hanchate, $P h D^{3}$
}

'Department of Medicine, Boston Medical Center, Boston University School of Medicine, Boston, MA, USA; ${ }^{2}$ RAND Corporation, 20 Park Plaza \#920, Boston, MA, USA; ${ }^{3}$ Department of Social Sciences and Health Policy, Wake Forest School of Medicine, Winston-Salem, NC, USA.

BACKGROUND: Little is known about the risk of admission for emergency department (ED) visits for ambulatory care sensitive conditions (ACSCs) by limited English proficient (LEP) patients.

OBJECTIVE: Estimate admission rates from ED for ACSCs comparing LEP and English proficient (EP) patients and examine how these rates vary at hospitals with a high versus low proportion of LEP patients.

DESIGN: Retrospective cohort study of California's 2017 inpatient and ED administrative data

PARTICIPANTS: Community-dwelling individuals $\geq 18$ years without a primary diagnosis of pregnancy or childbirth. LEP patients had a principal language other than English.

MAIN MEASURES: We used a series of linear probability models with incremental sets of covariates, including patient demographics, primary diagnosis, and Elixhauser comorbidities, to examine admission rate for visits of LEP versus EP patients. We then added an interaction covariate for high versus low LEP-serving hospital. We estimated models with and without hospital-level random effects.

KEY RESULTS: These analyses included 9,641,689 ED visits; $14.7 \%$ were for LEP patients. . Observed rate of admission for all ACSC ED visits was higher for LEP than for EP patients (26.2\% vs. 25.2; $p$ value <.001). Adjusted rate of admission was not statistically significant $27.3 \%$ [95\% CI 25.4-29.3\%] vs. 26.2\% [95\% CI 24.3-28.1\%]). For COPD, the difference was significant (36.8\% [95\% CI 35.0-38.6\%] vs. 33.3\% [95\% CI 31.7-34.9\%]). Difference in adjusted admission rate for LEP versus EP visits did not differ in high versus low LEP-serving hospitals.

CONCLUSIONS: In adjusted analyses, LEP was not a risk factor for admission for most ACSCs. This finding was observed in both high and low LEP-serving hospitals.

KEY WORDS: limited English proficiency; health disparities; ambulatory care sensitive conditions.

J Gen Intern Med 36(9):2683-91

DOI: $10.1007 / \mathrm{s} 11606-020-06523-5$

(c) Society of General Internal Medicine 2021

Received August 2, 2020

Accepted December 20, 2020

Published online February 2, 2021

\section{INTRODUCTION}

Twenty-five million people - $8 \%$ of the US population - has limited English proficiency (LEP). Patients with LEP are more likely to be uninsured and are less likely to have access to regular primary care. ${ }^{1,2}$ The lack of an adequate healthcare workforce with language skills to meet the needs of patients and underutilization of professional interpreters despite federal mandates likely contribute to compromise in communication between clinicians and patients. ${ }^{3-5}$ Patients with LEP are more likely to report poor understanding of their medical care, lower satisfaction with care, and difficulty getting health information and advice over the phone. ${ }^{6-9}$ Consequently, patients with LEP may experience worse care.

LEP patients may have higher emergency department (ED) utilization even when empaneled in a primary care practice. ${ }^{10}$ They are more likely to be admitted than English proficient (EP) patients presenting with similar conditions. ${ }^{10-13}$ Much of this evidence comes from single-center studies. Additionally, few studies have examined if LEP patients have more preventable hospitalizations than EP patients. ${ }^{14}$

Admission for an ambulatory care sensitive condition (ACSC) is a quality metric used to identify potentially preventable admissions. ${ }^{15}$ Although this metric has been examined in regard to race/ethnicity and insurance status, few studies have examined if LEP patients are at increased risk for admission for ACSCs compared to English proficient patients. ${ }^{14}$ One study using 2013-2014 data from New Jersey found that LEP patients were less likely to be admitted for ACSCs as compared to English proficient patients. ${ }^{14}$ The extent to which this pattern generalizes to other states is unknown. Previous studies have not examined the impact of hospital factors on admission. The quality of services to support the needs of LEP patients, such as robust interpreter services, may impact admission rates. The availability of these services may be related to the volume of LEP patients at a hospital.

California has the highest proportion of residents with LEP of any state $(19 \%) .{ }^{16,17}$ We used data from California to examine if the hospital admission rate for LEP patients differed from that for EP patients overall and for ACSCs. We additionally sought to examine if the disparity in admission 
rate between LEP and EP patients varied between hospitals that serve a higher versus lower percentage of LEP patients.

\section{METHODS}

\section{Data source}

Our primary data sources were California's 2017 ED and inpatient discharge databases. ${ }^{18,19} \mathrm{We}$ included data on hospital characteristics from the American Hospital Association (AHA) Annual Survey.

\section{Study Sample}

Our primary unit of analysis was an ED visit resulting in admission or discharge. We included ED visits to AHAidentified community hospitals for people aged $\geq 18$ years whose presentation to an ED resulted in either a hospital admission or discharge. ED visits or admissions with a primary diagnosis of "pregnancy or childbirth or diseases occurring in the perinatal period" were also excluded as these patients are not included in analyses of potentially preventable hospitalizations. We also excluded individuals for whom key variables (e.g., principal diagnosis, preferred language) were missing. Hospitals with fewer than $100 \mathrm{ED}$ visits, admission rate > $50 \%$, less than $1 \%$ of an LEP population, or fewer than 200 LEP patient visits were also excluded to improve generalizability and model stability (Fig. 1).

\section{Primary Exposure of Interest}

Any individual who selected a language other than English as the principal language used in communicating with healthcare providers was considered LEP. ${ }^{14,20,21}$ Those who selected English were considered English proficient. We further categorized LEP based on the most frequent languages in the dataset: Spanish, Chinese (Mandarin and Cantonese), Taga$\log$, Vietnamese, and other.

\section{Outcomes of Interest}

Our primary outcome was the proportion of ED visits resulting in admission. Our secondary outcome was the rate of admission for ACSCs. ${ }^{15}$ We used discharge diagnosis codes (ICD-10-CM) and the Agency for Healthcare Research and Quality (AHRQ)'s Prevention Quality Indicators algorithm to identify ACSCs. ${ }^{15}$ Specifically, we included admissions for ten conditions: diabetes with short-term complications, diabetes with long-term complications, uncontrolled diabetes without complications, chronic obstructive pulmonary disease, asthma, hypertension, heart failure, dehydration, bacterial pneumonia, or urinary tract infection. We did not include the admissions for amputation as this would always require inpatient admission. We categorized ACSCs into the following: (a) any ACSC; (b) acute
ACSCs (dehydration, bacterial pneumonia, and urinary tract infection); and (c) chronic ACSCs (short-term and long-term complications of diabetes, chronic obstructive lung disease or asthma in older adults, hypertension, heart failure, uncontrolled diabetes, and asthma in younger adults). ${ }^{15} \mathrm{We}$ also examined admission rates for each individual ACSC.

\section{Covariates}

Covariates included individual sex, age (18-30, 21-40, 4150, 51-64, and $\geq 65$ ), and race/ethnicity (Latino, non-Latino white, non-Latino Black, non-Latino Asian, and other). Those with unknown or missing race/ethnicity were categorized as "other." As socioeconomic indicators, we used patient's primary payor (commercial, Medicare, Medicaid, or other public insurance, self-pay, and other) and zip code median income for patient's zip code of residence. ${ }^{22} \mathrm{We}$ additionally included clinical characteristics that may influence admission decision: primary diagnosis, classified using ICD-10-CM Clinical Classification Software (CCS), and Elixhauser comorbidities. ${ }^{23,24}$

We included hospital-level characteristics: teaching status (yes/no), ownership (public, private for-profit, and private non-profit), hospital bed size (6-199 beds, 200-399, $\geq 400$ beds), and safety net hospital (yes/no), which we defined as the top quartile of hospitals in California by proportion of patients covered by Medicaid. We grouped all hospitals into deciles by the proportion of LEP ED visits and defined the hospitals in the 60th percentile or greater as "high LEPserving."

\section{Statistical Analyses}

We performed chi-square and Student's $t$ tests for unadjusted analyses comparing visits for LEP and EP patients. We clustered patients at the hospital level and calculated the difference in admission rate overall and for ACSCs for LEP and EP patient visits. To obtain adjusted admission rates for LEP and EP patient visits, we estimated linear probability regression models with individual ED visit as the unit of observation and dichotomous indicator of admission as the outcome. We estimated a series of models, incrementally adding covariates, to enable better interpretation of the role of different covariate domains. First, we adjusted for age, sex, race/ethnicity, primary discharge diagnosis, and Elixhauser comorbidities. We then added insurance type, zip code income quartile, and patient county-level fixed effects. Finally, we included hospital characteristics, and to adjust for clustering at specific hospitals, we used random effects. For all models, we obtained standard errors clustered at the hospital level. ${ }^{25}$ These models were estimated separately for all ED visits and subgroups of ACSCs. Adding interaction terms for high and low LEPserving hospitals to the linear probability regression models, we estimated the difference in adjusted admission rates between LEP and EP patients in high LEP-serving versus low LEP-serving hospitals. 
Community dwelling individuals $\geq 18$ years of age with an ED visit that resulted in discharge or admission to the hospital and for whom the primary diagnosis was not pregnancy or childbirth $n=11,557,283$

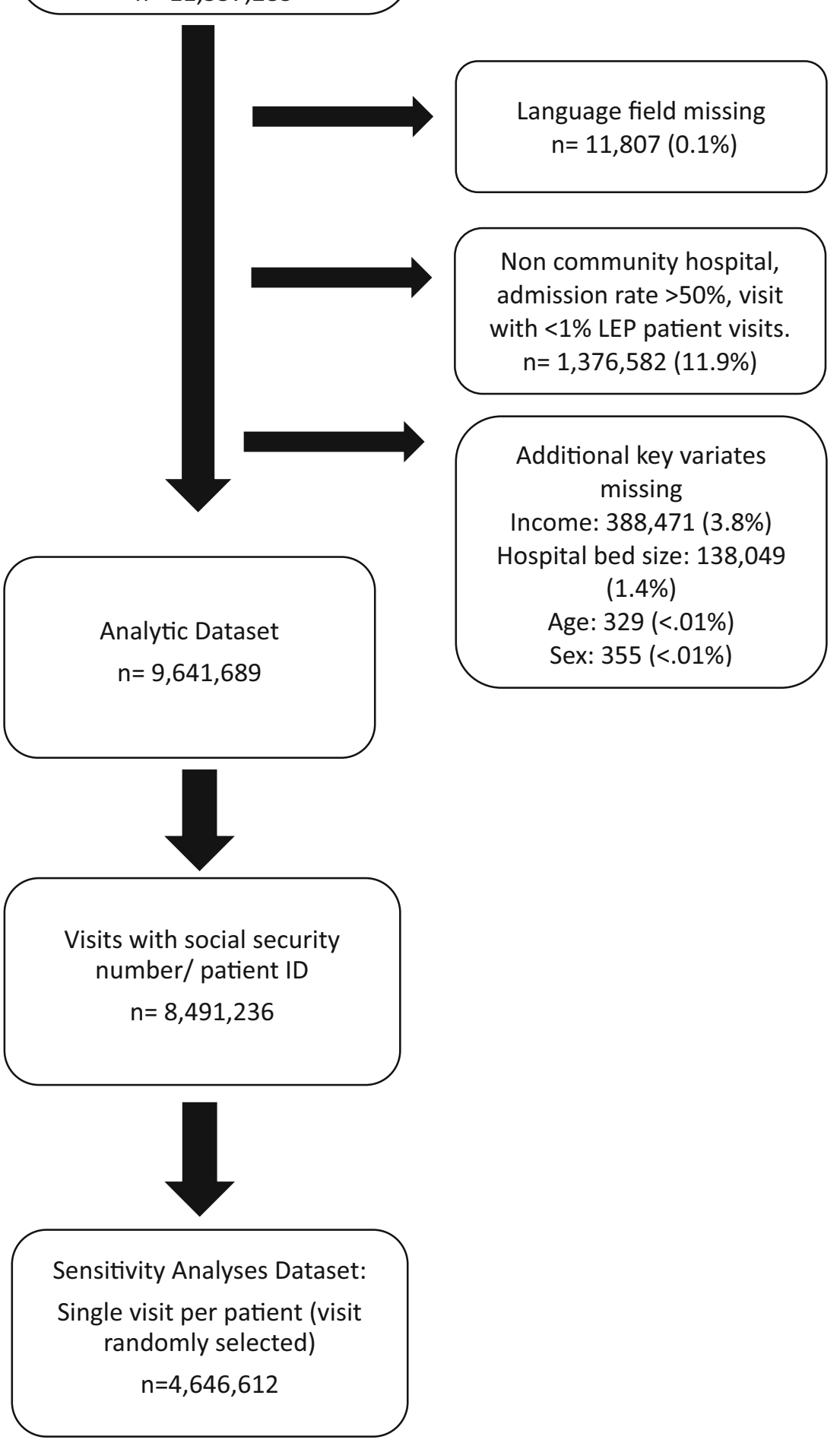

Figure 1 Analytic dataset and sensitivity analyses. 
Table 1 Sample Characteristics by LEP and English Proficiency

\begin{tabular}{|c|c|c|c|}
\hline$\overline{\text { Variable }}$ & $\begin{array}{l}\text { LEP } \\
n=1,421,385\end{array}$ & $\begin{array}{l}\mathrm{EP} \\
n=8,220,304\end{array}$ & $p$ value \\
\hline Female & $58.8 \%$ & $53 . \%$ & $<.0001$ \\
\hline Age category & & & $<.0001$ \\
\hline $18-30$ & $9.28 \%$ & $23.09 \%$ & \\
\hline $31-40$ & $14.22 \%$ & $16.45 \%$ & \\
\hline $41-50$ & $18.30 \%$ & $13.99 \%$ & \\
\hline $51-64$ & $26.86 \%$ & $22.52 \%$ & \\
\hline$\geq 65$ & $31.34 \%$ & $23.95 \%$ & \\
\hline Äge mean (ST) & $54.7(18.7)$ & $48.7(20.5)$ & $<.0001$ \\
\hline Race/ethnicity & & & $<.0001$ \\
\hline White & $6.0 \%$ & $46.4 \%$ & \\
\hline African-American & $0.3 \%$ & $13.2 \%$ & \\
\hline Latino & $76.0 \%$ & $27.6 \%$ & \\
\hline Asian & $10.8 \%$ & $5.1 \%$ & \\
\hline Other/unknown/missing & $6.9 \%$ & $7.8 \%$ & \\
\hline Language & & & N/A \\
\hline English & N/A & $100 \%$ & \\
\hline Spanish & $80.2 \%$ & N/A & \\
\hline Vietnamese & $2.7 \%$ & N/A & \\
\hline Mandarin/Cantonese & $2.0 \%$ & N/A & \\
\hline Tagalog & $1.4 \%$ & N/A & \\
\hline Other & $13.6 \%$ & N/A & \\
\hline Insurance & & & $<.0001$ \\
\hline Commercial & $13.8 \%$ & $26.6 \%$ & \\
\hline Medicaid & $47.6 \%$ & $35.7 \%$ & \\
\hline Medicare & $27.5 \%$ & $28.1 \%$ & \\
\hline Other & $2.8 \%$ & $3.4 \%$ & \\
\hline Self-pay & $8.4 \%$ & $6.1 \%$ & \\
\hline Zip code income quartiles (lowest to highest) & & & $<.0001$ \\
\hline $1 \mathrm{st}$ & $33.9 \%$ & $24.3 \%$ & \\
\hline 2nd & $28.7 \%$ & $24.0 \%$ & \\
\hline $3 \mathrm{rd}$ & $22.7 \%$ & $25.4 \%$ & \\
\hline 4th & $14.8 \%$ & $26.4 \%$ & \\
\hline Hospital ownership & & & $<.0001$ \\
\hline Government non-federal & $26.7 \%$ & $17.5 \%$ & \\
\hline Non-government not for profit & $60.6 \%$ & $69.2 \%$ & \\
\hline Investor owned (for profit) & $12.7 \%$ & $13.3 \%$ & \\
\hline Teaching hospital & & & $<.0001$ \\
\hline Yes & $12.2 \%$ & $8.1 \%$ & \\
\hline Safety net hospital & & & $<.0001$ \\
\hline Yes & $29.9 \%$ & $22.8 \%$ & $<.0001$ \\
\hline Hospital size & & & $<.0001$ \\
\hline $6-199$ beds & $34.3 \%$ & $34.1 \%$ & \\
\hline $200-399$ beds & $44.0 \%$ & $45.8 \%$ & \\
\hline$\geq 400$ beds & $21.8 \%$ & $20.1 \%$ & \\
\hline$\overline{\text { Admission rate }}$ & $26.2 \%$ & $25.2 \%$ & $<.0001$ \\
\hline Admission rate by ACSC & & & \\
\hline Overall & $18.9 \%$ & $16.1 \%$ & $<.0001$ \\
\hline Acute condition & $18.7 \%$ & $18.1 \%$ & $<.0001$ \\
\hline Chronic condition & $34.5 \%$ & $32.2 \%$ & $<.0001$ \\
\hline Diabetes short-term complications & $54.0 \%$ & $70.9 \%$ & $<.0001$ \\
\hline Diabetes long-term complications & $54.4 \%$ & $54.0 \%$ & 0.46 \\
\hline COPD or asthma in older adults & $37.8 \%$ & $31.8 \%$ & $<.0001$ \\
\hline Hypertension & $13.9 \%$ & $11.7 \%$ & $<.0001$ \\
\hline Heart failure & $72.1 \%$ & $66.2 \%$ & $<.0001$ \\
\hline Dehydration & $48.7 \%$ & $39.7 \%$ & $<.0001$ \\
\hline Community-acquired pneumonia & $34.1 \%$ & $30.1 \%$ & $<.0001$ \\
\hline Urinary tract infection & $8.9 \%$ & $8.3 \%$ & $<.0001$ \\
\hline Uncontrolled diabetes & $16.4 \%$ & $16.1 \%$ & 0.39 \\
\hline Asthma in younger adult & $33.7 \%$ & $17.9 \%$ & $<.0001$ \\
\hline
\end{tabular}

LEP, limited English proficient; EP, English proficient; ACSC, ambulatory care sensitive condition

\section{Sensitivity Analyses}

We examined if repeated ED visits across individuals affected our estimates. For this, we selected the subgroup of observations with a valid patient ID $(n=8,615,842,89.4 \%)$. Among the subgroup of patients with two or more ED visits, we randomly selected one visit per patient. We estimated the linear probability models for this subgroup of observations. We also examined sensitivity of our estimates to the choice of cutoff in defining high and low LEP-serving hospitals; we examined alternative cutoffs of 70th percentile and 50th percentile. Analyses were performed using SAS (version 9.4) and Stata (version 15.1). This study was approved by the IRB at Boston University School of Medicine. 


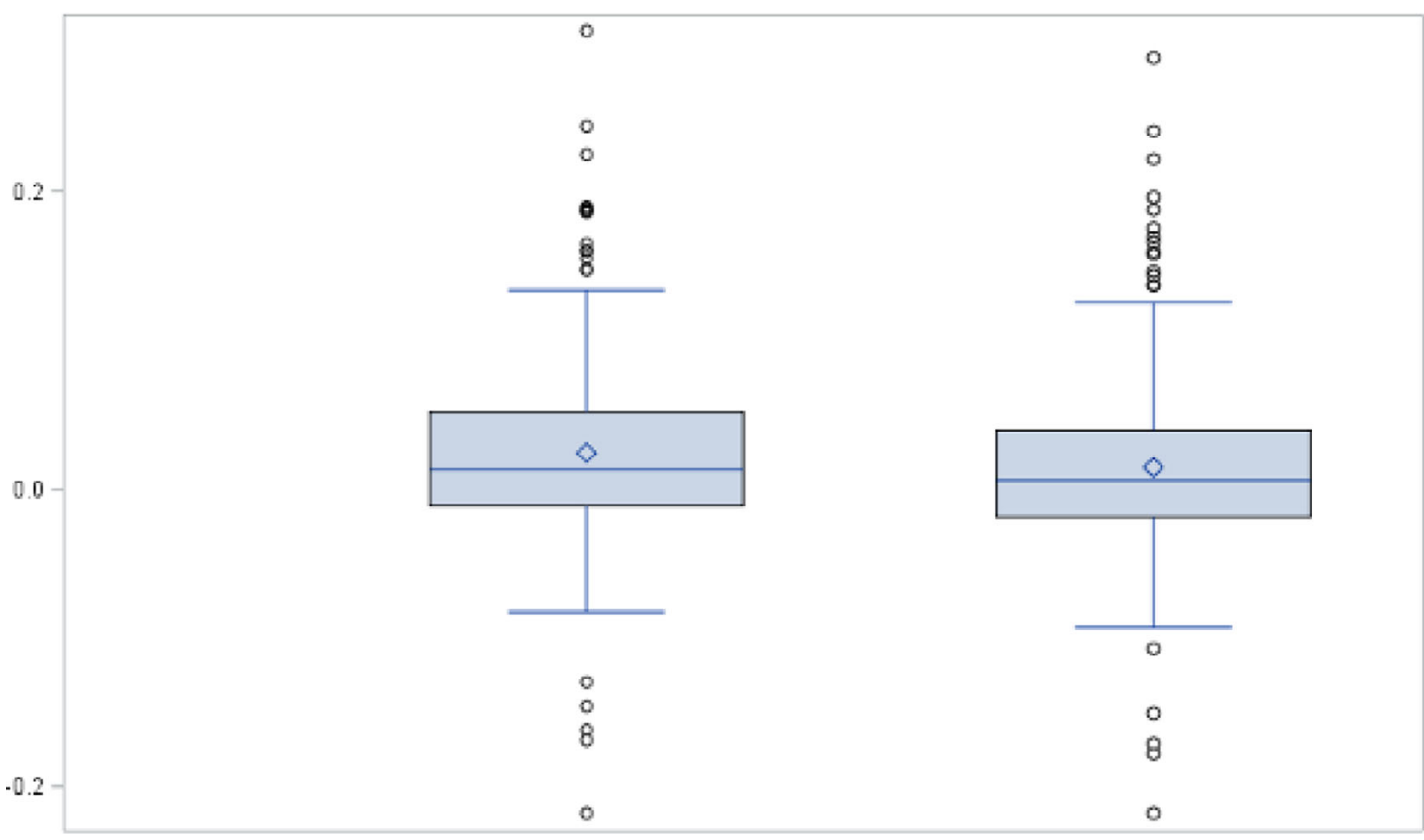

Any admission

ACSC admission

Figure 2 Distribution of difference in admission rate at each hospital, between limited English proficient patient visits and English proficient patient visits: any admission and ACSC admissions.

Table 2 Adjusted Emergency Department Encounter Admission Rates for Visits of Limited English Proficient Patients Versus English Proficient Patient Visits, Overall and for Ambulatory Care Sensitive Conditions

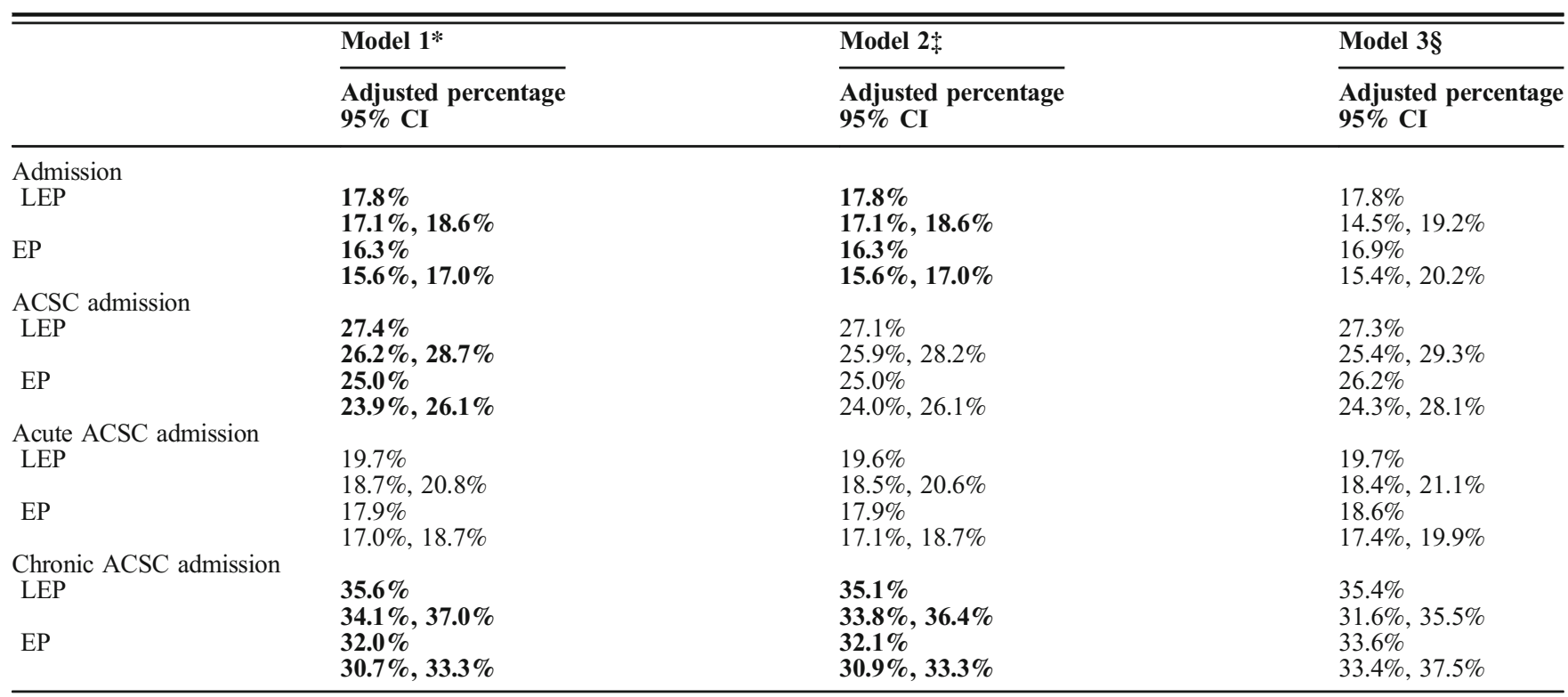

Bold indicates statistically significant at a level of $p<.05$

LEP, limited English proficient; EP, English proficient; ACSC, ambulatory care sensitive condition

*Model 1: age, sex, race, primary diagnosis, Elixhauser comorbidities, with standard errors clustered at hospital level. In models of ACSCs, primary diagnosis was not included

\$Model 2: Model 1 and insurance type, quarter, zip code income quartile, patient county of residence, with standard errors clustered at the hospital level. In models of ACSCs, primary diagnosis was not included

\$Model 3: Model 2 and hospital teaching status, hospital ownership, hospital random effects, and with standard errors clustered at the hospital level. In models of ACSCs, primary diagnosis was not included 
Table 3 Adjusted Emergency Department Encounter Admission Rates for Visits of Limited English Proficient Patients Versus English Proficient Patient Visits for Specific Ambulatory Care Sensitive Conditions

\begin{tabular}{|c|c|}
\hline \multirow[t]{2}{*}{$\overline{\mathrm{ACSC}}$} & Model 3* \\
\hline & $\begin{array}{l}\text { Adjusted percentage } \\
95 \% \mathrm{CI}\end{array}$ \\
\hline
\end{tabular}

Diabetes short-term complication

\section{LEP}

EP

Diabetes long-term complication LEP

EP

COPD or asthma in older adults LEP

EP

Hypertension

LEP

EP

Heart failure

LEP

EP

Dehydration

LEP

EP

Community-acquired pneumonia LEP

EP

Urinary tract infection

LEP

EP

LEP

EP

Asthma in younger adult

LEP

EP
Uncontrolled diabetes
$69.7 \%$

$68.9 \%, 72.4 \%$

$70.6 \%$

$67.1 \%, 72.3 \%$

$55.8 \%$

$54.0 \%, 57.6 \%$

$54.5 \%$

$52.8 \%, 56.3 \%$

$36.8 \%$

$35.0 \%, 38.6 \%$

$33.3 \%$

$31.7 \%, 34.9 \%$

$13.7 \%$

$12.8 \%, 14.7 \%$

$12.2 \%$

$11.5 \%, 13.0 \%$

$69.8 \%$

$67.6 \%, 72.0 \%$

$67.1 \%$

$65.1 \%, 69.0 \%$

$42.6 \%$

$40.8 \%, 44.3 \%$

$41.6 \%$

$40.0 \%, 43.1 \%$

$33.2 \%$

$31.5 \%, 35.0 \%$

$31.2 \%$

$29.7 \%, 32.6 \%$

$9.5 \%$

$8.9 \%$

$8.0 \%, 9.7 \%$

$18.8 \%$

$17.4 \%, 20.2 \%$

$17.5 \%$,

$16.3 \%, 18.7 \%$

$19.4 \%$

$18.3 \%, 20.5 \%$

$19.4 \%$ \%

$18.9 \%, 19.9 \%$
$8.6 \%, 10.4 \%$

Bold indicates statistically significant at a level of $p<.05$.

LEP, limited English proficient; EP, English proficient; ACSC, ambulatory care sensitive condition

*Model 3: Model 2 and hospital teaching status, hospital ownership, hospital random effects, and with standard errors clustered at the hospital level. Primary diagnosis not included in models with ACSCs

\section{RESULTS}

There were 9,641,689 ED visits included in our sample, of which $14.7 \%$ were for patients with LEP (Table 1). LEP patients were more likely to be female $(58.8 \%$ vs. $53.9 \%, p<$ .0001 ), older (mean age 54.7 vs. $48.7, p<.0001$ ), and more likely to identify as Latino $(76.0 \%$ vs. $27.6 \%, p<.0001)$. Those with LEP were more likely to have Medicaid insurance
Table 4 Differences in Emergency Department Encounter Admission Rates for Limited English Proficient Versus English Proficient Patients in High Versus Low Limited English ProficientServing Hospitals

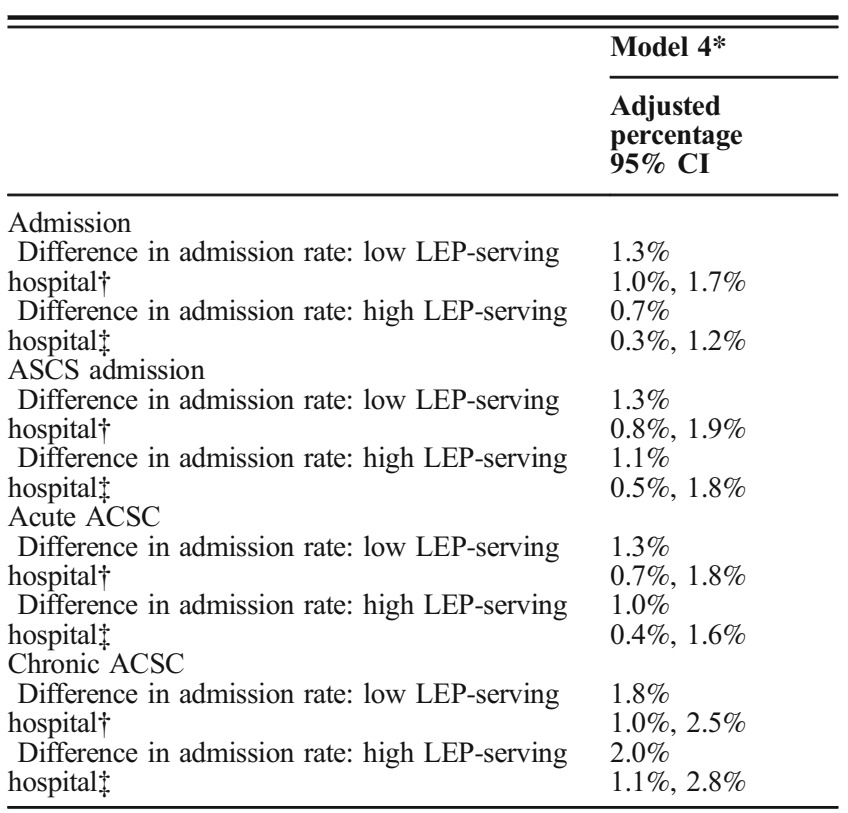

LEP, limited English proficient; ACSC, ambulatory care sensitive condition

*Adjusting for age, sex, race, insurance type, quarter, zip code income quartile, clinical classification software primary diagnosis, Elixhauser comorbidities, hospital teaching status, hospital ownership. LEP patient at low LEP-serving hospital is referent. Standard errors are clustered at the hospital level. In models with ACSCs, primary diagnosis not included

†A low LEP-serving hospital is one in which fewer than $20 \%$ of visits are for LEP patients, which is also hospitals below the 6th decile for proportion of visits where the patient has LEP

$\mp$ A high LEP-serving hospital is one in which $20 \%$ or greater of visits are for LEP patients, which is also hospitals in the 6th decile or above for proportion of visits where the patient has LEP

(47.6\% vs. $35.7 \%, p$ value $<.0001)$. They were also more likely to reside in a zip code in the lowest income quartile $(13.8 \%$ vs. $26.6 \%, p$ value $<.0001)$, and more likely to have a visit at a safety net hospital $(29.9 \%$ vs. $22.8 \%, p<.0001)$.

In unadjusted analyses, visits for LEP patients were more likely to result in an admission overall, for any ACSC, for any acute ACSC, and for any chronic ACSC (26.2\% vs. 25.2 ; $18.9 \%$ vs. $16.1 \% ; 18.7 \%$ vs. $18.1 \% ; 34.5 \%$ vs. $32.2 \%$, all $p$ values $<.0001$ ) (Table 1 ). LEP patients were more likely to be admitted for COPD or asthma in an older adult and hypertension, heart failure, dehydration, community-acquired pneumonia, urinary tract infections, and asthma in a younger adult. LEP patients were less likely to be admitted for diabetes with short-term complications $(54.0 \%$ vs. $70.9 \%, p<.0001)$ (Table 1). Hospitals varied in their disparity in admission rates for LEP and EP patient visits. The median difference in hospital-level admission rate was $1.3 \%$ (i.e., more LEP patients were admitted than EP patients) with an inter-quartile range (IQR) of -1.1 to $5.1 \%$. For ACSCs, the median difference was $-1.1 \%$ (i.e., fewer LEP patients were admitted than EP patients) with an IQR of -4.6 to $3.3 \%$ (Fig. 2). 
Table 2 reports the adjusted rate of admission for LEP and EP patients. LEP patients were more likely to be admitted for any condition (17.8\% [95\% CI 17.1-18.6\%] vs. $16.3 \%$ [95\% CI 15.6-17.0\%]), but this difference was no longer statistically significant when adjusted for hospital-level clustering (model 3). LEP patients were also more likely to be admitted for any ACSC $(27.4 \%$ [95\% CI $26.2-28.7 \%$ ] vs. $25.0 \%$ [95\% CI 23.9-26.1\%]) but this difference was no longer significant when insurance type, visit quarter, patient county fixed effects, and zip code income quartile were added to the model (model 2). There was no significant difference in admission for acute ACSCs (models 1-3). LEP patients were more likely to be admitted for chronic ACSCs (35.6\% [95\% CI 34.1-37.0\%] vs. $32.0 \%$ [95\% CI 30.7-33.3\%]), but this difference was no longer statistically significant when hospital-level factors were added to the model (model 3).

Evaluated for individual ACSCs, LEP patients were more likely to be admitted for COPD or asthma in older adults and this finding was significant in all models $(36.8 \%$ [95\% CI $35.0-38.6 \%$ ] vs. $33.3 \%$ [95\% CI 31.7-34.9\%]). For none of the other ACSCs was the estimated difference in likelihood of admission between LEP and EP patients statistically significant in the fully adjusted models (Table 3 ).

Table 4 gives estimated difference in the admission rate for LEP versus EP patients across hospitals grouped as high and low LEP-serving. The estimated difference in admission rate in low LEP-serving hospitals was $1.3 \%$ (95\% CI 1.0-1.7\%) versus $0.7 \%$ (95\% CI $0.3-1.2 \%$ ) in high LEP-serving hospitals. For ACSCs, the differences in admission rates for low and high LEP-serving hospitals were similar.

Analyses of specific languages demonstrated variable admission rates for chronic ACSCs, short-term complications for diabetes, long-term complications for diabetes, COPD, hypertension, urinary tract infections, and uncontrolled diabetes. Admission rates for those who spoke Mandarin/Cantonese, those who spoke Vietnamese, and those whose language was categorized as "other" were the language groups for which there was a significant difference in admission rate compared to English (Appendix Table 1). Adjusting the cutoff for LEPserving hospital did not alter results in any meaningful way (Appendix Tables 2 and 3). Sensitivity analyses among those with a valid patient ID and at the individual patient level were consistent with the overall analysis (Appendix Tables 4 and 5).

\section{DISCUSSION}

Using California statewide data, we found that older adult LEP patients presenting with COPD or asthma were more likely to be admitted than EP patients presenting with the same diagnosis codes even after accounting for patient and hospital factors. Patients with LEP were more likely to be admitted to the hospital than EP patients for any admission, for any ACSC, and for chronic ACSCs. However, the difference in admission rate for acute ACSCs was not significant when adjusted for socioeconomic factors. The difference between LEP and EP patients in overall admission rates and admission for most chronic ACSCs was not significant when adjusted for hospital factors, indicating that differences in the hospitals where LEP and EP patients received care may be associated with the unadjusted (observed) differences in admission rates.

Counter to our hypothesis, there was no significant difference in admission rates in the fully adjusted models for most ACSCs, and in cases where there was a difference, the difference was quite small. It is possible that data on principal language did not adequately capture those who experience language barriers in healthcare. An individual may be proficient in English but report another principal language and this would bias the results towards the null. Alternatively, compared to other states, California may be providing more effective care to their LEP population than other states with a high proportion of LEP residents. California has made concerted efforts at the state level to address the care of LEP patients. In 2005, California developed the healthcare interpreter network to provide video and voice interpretation to public hospitals and clinics that serve the California Medicaid population. ${ }^{26}$ The Health Care Language Assistance Act which went into full effect in 2009 holds health plans accountable for the provision of language services and requires health plans and health insurers to provide their enrollees with interpreter services and translated material. ${ }^{27}$

Accordingly, state-specific policies may explain why our results differed from those of Anderson et al. who evaluated the impact of language and admission for ACSCs in New Jersey and found a lower admission rate for those with LEP. ${ }^{14}$ They hypothesized LEP patients may be more likely to turn to the ED for non-urgent primary care issues while EP patients would first seek outpatient care and only present to the ED for more severe issues. While both studies controlled for insurance, it is possible that Californians with LEP have, on average, more established connections in primary care than those in New Jersey and that this resulted in minimal difference in admission for acute ACSCs between LEP and EP patients in California but persistent differences for some chronic ACSCs. ${ }^{14}$ Although these differences were quite small in percentage terms, they represent large spending given the high volumes and high cost of an ED visit $(\$ 1106)$ and inpatient stay $(\$ 14,892) .{ }^{28}$ The differences in admission rates for chronic ACSCs are consistent with prior literature demonstrating poorer chronic disease management for those with LEP even with access to primary care. ${ }^{29-31}$ This finding may reflect factors beyond access to care such as health literacy, ability to afford medications, or difficulty understanding provider's instructions around disease management due to language barriers. Our findings may also differ from those of Anderson et al. because the predominant languages in New Jersey differ from those in California; distinct sociolinguistic contexts may influence cultural differences in how people interact with the healthcare system. 
Contrary to our hypothesis, hospitals serving a higher portion of LEP patients did not do better than hospitals serving a lower portion of LEP patients. One explanation for this finding may be that having a high proportion of LEP patients does not necessarily result in improved care for this population. These hospitals may not be overcoming language barriers possibly due to a shortage of interpreters for the high demand or underutilization of interpreters for other reasons. ${ }^{5,32-34}$ The range in disparity in admission between hospitals warrants further research to understand what other hospital characteristics contribute to disparities in admission rates between LEP and EP patients. Our study also demonstrates that a major driver of difference in admission rates relates to which hospital LEP and EP patients present. Further research is needed to understand what factors lead LEP and EP patients to different hospitals.

We also found that admission rates for some ACSCs varied by specific language. One reason for this difference may be the linguistic capabilities of providers, as language-concordant care has been shown to improve outcomes. ${ }^{35-39}$ About 30\% of physicians in California speak Spanish while only 3\% speak Cantonese, 6\% Mandarin, 6\% Tagalog, and 4\% Vietnamese. ${ }^{3}$ Some of the variability by language may be a reflection of sociocultural differences in conception of illness and self-care; such factors can influence when patients present to emergency departments, how they describe their symptoms, and the likelihood of admission. ${ }^{40,41}$

Our study has several limitations. First, it may be inappropriate to extrapolate our findings to other states where the predominant languages may differ from those of California and where state policies may differ. Second, although we were able to control for comorbidities, which may influence risk of admission, we were unable to control for acuity at presentation. Additionally, we were not able to measure characteristics of patients' primary care environments. Third, because of the high proportion of LEP patients without a person-level indicator, we are unable to adjust for person-level clustering of ED visits. However, our subgroup analysis for people with personlevel indicators exhibited consistent results. Fourth, our indicators for income were area level and therefore may not accurately represent the socioeconomic status of the individual patient. Additionally, we were not able to identify dual enrolled (Medicare/Medicaid) individuals. Finally, principal language as captured by these data cannot be confirmed; it is possible that some individuals who were identified as LEP in fact spoke English and some who identified as English speaking were in fact LEP. ${ }^{42}$

Using data from California, we found that patients with LEP are at a small but significant increased risk for admission from the emergency department for COPD or asthma in older adults but no other ACSCs in fully adjusted models. The disparity in admission rate between LEP and EP patients was not different between hospitals that serve higher versus lower proportions of LEP patients. More research is needed to understand what factors impact differences in admission rates for ACSCs between LEP and EP patients.

Supplementary Information The online version contains supplementary material available at https://doi.org/10.1007/s11606-02006523-5.

Acknowledgments: The authors would like to acknowledge Lisa Marks for her technical support.

Corresponding Author: Lucy Schulson, MD, MPH; RAND Corporation, 20 Park Plaza \#920, Boston, MA 02116, USA (e-mail: schulson@rand.org).

Funding This work was supported in part by a Health Resources and Services Administration's National Research Service Award (T32HP10028) (Dr. Schulson), Agency for Healthcare Research and Quality Award (T32HSO22242) (Dr. Lin), and National Institute on Minority Health and Health Disparities Grant R01MD011594 (Dr. Hanchate).

\section{Compliance with Ethical Standards:}

Conflict of Interest: The authors declare that they do not have a conflict of interest.

\section{REFERENCES}

1. Lu T, Myerson R. Disparities in Health Insurance Coverage and Access to Care by English Language Proficiency in the USA, 2006-2016. J Gen Intern Med. 2020. https://doi.org/10.1007/s11606-019-05609-Z

2. DuBard CA, Gizlice $Z$. Language spoken and differences in health status, access to care, and receipt of preventive services among US Hispanics. Am J Public Health 2008;98(11):2021-2028. https://doi.org/10.2105/ AJPH.2007.119008

3. Garcia $\mathrm{ME}$, Bindman $\mathrm{AB}$, Coffman J. Language-Concordant Primary Care Physicians for a Diverse Population: The View from California. Health Equity 2019;3(1):343-349. https://doi.org/10.1089/heq.2019. 0035

4. Diamond LC, Tuot DS, Karliner LS. The Use of Spanish Language Skills by Physicians and Nurses: Policy Implications for Teaching and Testing. J Gen Intern Med 2012;27(1):117-123. https://doi.org/10.1007/s11606011-1779-5

5. Schenker Y, Pérez-Stable EJ, Nickleach D, Karliner LS. Patterns of interpreter use for hospitalized patients with limited English proficiency. J Gen Intern Med 2011;26(7):712-717. https://doi.org/10.1007/ s11606-010-1619-Z

6. Calo WA, Cubillos L, Breen J, et al. Experiences of Latinos with limited English proficiency with patient registration systems and their interactions with clinic front office staff: an exploratory study to inform community-based translational research in North Carolina. BMC Health Serv Res. 2015;15(1). 10.1186/s12913-015-1235-Z

7. Regan Freeman R. Primary care for limited English-speaking patients and parents. J Am Assoc Nurse Pract 2015;27(3):167-176. https://doi. org/10.1002/2327-6924.12148

8. Morales LS, Cunningham WE, Brown JA, Liu H, Hays RD. Are Latinos less satisfied with communication by health care providers? J Gen Intern Med 1999;14(7):409-417.

9. Pippins JR, Alegria M, Haas JS. Association between language proficiency and the quality of primary care among a national sample of insured Latinos. Med Care 2007;45(11):1020-1025. https://doi.org/10.1097/ MLR.0b013e31814847be

10. Njeru JW, St Sauver JL, Jacobson DJ, et al. Emergency department and inpatient health care utilization among patients who require interpreter services. BMC Health Serv Res 2015;15:214. https://doi.org/10.1186/ s12913-015-0874-4

11. Schulson L, Novack V, Smulowitz PB, Dechen T, Landon BE. Emergency Department Care for Patients with Limited English Proficiency: a 
Retrospective Cohort Study. J Gen Intern Med Published online September 5, 2018. https://doi.org/10.1007/s11606-018-4493-8

12. Gallagher RA, Porter S, Monuteaux MC, Stack AM. Unscheduled return visits to the emergency department: the impact of language. Pediatr Emerg Care 2013;29(5):579-583. https://doi.org/10.1097/PEC. Ob013e31828e62f4

13. Hampers LC, Cha S, Gutglass DJ, Binns HJ, Krug SE. Language barriers and resource utilization in a pediatric emergency department. Pediatrics. 1999; 103(6 Pt 1):1253-1256.

14. Anderson TS, Karliner LS, Lin GA. Association of Primary Language and Hospitalization for Ambulatory Care Sensitive Conditions. Med Care 2020;58(1):45-51. https://doi.org/10.1097/MLR.0000000000001245

15. Agency for Healthcare Research and Quality. Prevention quality indicators overview. 2018. https://www.qualityindicators.ahrq.gov/modules/ pqi_resources.aspx

16. Zong J, Batalova J. The Limited English Proficient Population in the United States. Migr Policy Inst Published online July 8, 2015. https:// www.migrationpolicy.org/article/limited-english-proficient-populationunited-states

17. American Community Survey. California Department of Finance Estimates and Projections; US Census Bureau Estimates; Decennial Censuses.; 2017.

18. California Emergency Department \& Ambulatory Surgery Data Reporting Manual. 5th ed. Office of Statewide Health Planning and Development; 2020. https://oshpd.ca.gov/data-and-reports/submit-data/patient-data/edas-reporting/

19. Inpatient Discharge Reporting. Office of Statewide Health Planning and Development; 2020. https://oshpd.ca.gov/data-and-reports/submit-data/patient-data/inpatient-reporting/

20. Biswas S, Dinh D, Lucas M, et al. Impact of limited English proficiency on presentation and clinical outcomes of patients undergoing primary percutaneous coronary intervention. Eur Heart J - Qual Care Clin Outcomes. Published online November 29, 2019:qcz061. 10.1093/ ehjqcco/qcz061

21. Hines A, Andrews R, Moy E, Barrett M, Coffey R. Disparities in Rates of Inpatient Mortality and Adverse Events: Race/Ethnicity and Language as Independent Contributors. Int $\mathrm{J}$ Environ Res Public Health 2014;11(12):13017-13034. https://doi.org/10.3390/ijerph111213017

22. American Community Survery 2012-2016. United States Census Bureau

23. Centers for Disease Control and Prevention. International Classification of Diseases, Tenth Revision, Clinical Modification (ICD-10-CM). Published online 2017. Accessed March 5, 2018. http://www.cdc.gov/nchs/icd/ icd $9 \mathrm{~cm}$. htm.

24. Elixhauser A, Steiner C, Harris DR, Coffey RM. Comorbidity measures for use with administrative data. Med Care 1998;36(1):8-27. https://doi org/10.1097/00005650-199801000-00004

25. Colin Cameron A, Miller DL. A Practitioner's Guide to Cluster-Robust Inference. J Hum Resour 2015;50(2):317-372. https://doi.org/10.3368/ jhr.50.2.317

26. Health Care Interpreter Network. Healthcare Interpreter Network: About Us. . http://www.hcin.org/

27. Escutia, Perata. The Health Care Language Access Act (SB 853).; 2003.

28. Access and Cost Trends, Medical Expenditure Panel Survey, 2017. Agency for Healthcare Research and Quality; 2017.

29. Wisnivesky JP, Krauskopf K, Wolf MS, et al. The association between language proficiency and outcomes of elderly patients with asthma. Ann
Allergy Asthma Immunol Off Publ Am Coll Allergy Asthma Immunol 2012;109(3):179-184. https://doi.org/10.1016/j.anai.2012.06.016

30. Kim EJ, Kim T, Paasche-Orlow MK, Rose AJ, Hanchate AD. Disparities in Hypertension Associated with Limited English Proficiency. J Gen Intern Med 2017;32(6):632-639. https://doi.org/10.1007/s11606-017-3999-9

31. Seman M, Karanatsios B, Simons K, et al. The impact of cultural and linguistic diversity on hospital readmission in patients hospitalized with acute heart failure. Eur Heart J - Qual Care Clin Outcomes. Published online July 23, 2019:qcz034. https://doi.org/10.1093/ehjqcco/qcz034

32. Mayo R, Parker VG, Sherrill WW, et al. Cutting Corners: Provider Perceptions of Interpretation Services and Factors Related to Use of an Ad Hoc Interpreter. Hisp Health Care Int 2016;14(2):73-80. https://doi. org/10.1177/1540415316646097

33. Hsieh E. Not just "getting by": factors influencing providers' choice of interpreters. J Gen Intern Med 2015;30(1):75-82. https://doi.org/10. 1007/s11606-014-3066-8

34. Ramirez D, Engel KG, Tang TS. Language interpreter utilization in the emergency department setting: a clinical review. J Health Care Poor Underserved 2008;19(2):352-362. https://doi.org/10.1353/hpu.0.0019

35. Fernandez A, Schillinger D, Warton EM, et al. Language barriers, physician-patient language concordance, and glycemic control among insured Latinos with diabetes: the Diabetes Study of Northern California (DISTANCE). J Gen Intern Med 2011;26(2):170-176. https://doi.org/10. 1007/s11606-010-1507-6

36. Ngo-Metzger Q, Sorkin DH, Phillips RS, et al. Providing high-quality care for limited English proficient patients: the importance of language concordance and interpreter use. J Gen Intern Med 2007;22 Suppl 2:324-330. https://doi.org/10.1007/s11606-007-0340-z

37. Villalobos BT, Bridges AJ, Anastasia EA, Ojeda CA, Rodriguez JH, Gomez D. Effects of language concordance and interpreter use on therapeutic alliance in Spanish-speaking integrated behavioral health care patients. Psychol Serv 2016;13(1):49-59. https://doi.org/10.1037/ser0000051

38. Green AR, Ngo-Metzger Q, Legedza ATR, Massagli MP, Phillips RS, Iezzoni LI. Interpreter services, language concordance, and health care quality. Experiences of Asian Americans with limited English proficiency. J Gen Intern Med 2005;20(11):1050-1056. https://doi.org/10.1111/j.15251497.2005.0223.x

39. Diamond L, Izquierdo K, Canfield D, Matsoukas K, Gany F. A Systematic Review of the Impact of Patient-Physician Non-English Language Concordance on Quality of Care and Outcomes. J Gen Intern Med 2019;34(8): 1591-1606. https://doi.org/10.1007/s11606-019-04847-5

40. Yeheskel A, Rawal S. Exploring the "Patient Experience" of Individuals with Limited English Proficiency: A Scoping Review. J Immigr Minor Health 2019;21(4):853-878. https://doi.org/10.1007/s10903-0180816-4

41. Shaw SJ, Huebner C, Armin J, Orzech K, Orzech K, Vivian J. The role of culture in health literacy and chronic disease screening and management. J Immigr Minor Health 2009;11(6):460-467. https://doi.org/10. 1007/s 10903-008-9135-5

42. Klinger EV, Carlini SV, Gonzalez I, et al. Accuracy of race, ethnicity, and language preference in an electronic health record. J Gen Intern Med 2015;30(6):719-723. https://doi.org/10.1007/s11606-014-3102-8

Publisher's Note: Springer Nature remains neutral with regard to jurisdictional claims in published maps and institutional affiliations. 